FACTA UNIVERSITATIS (NIŠ)

Ser. Math. Inform. Vol. 34, No 2 (2019), 341-357

https://doi.org/10.22190/FUMI1902341H

\title{
EXISTENCE AND STABILITY RESULTS FOR FRACTIONAL DIFFERENTIAL EQUATIONS WITH TWO CAPUTO FRACTIONAL DERIVATIVES
}

\author{
Mohamed Houas and Mohamed Bezziou
}

(C) 2019 by University of Niš, Serbia | Creative Commons Licence: CC BY-NC-ND

\begin{abstract}
In this paper, we discuss the existence, uniqueness and stability of solutions for a nonlocal boundary value problem of nonlinear fractional differential equations with two Caputo fractional derivatives. By applying the contraction mapping and O'Regan fixed point theorem, the existence results are obtained. We also derive the Ulam-Hyers stability of solutions. Finally, some examples are given to illustrate our results.

Keywords: Caputo derivative, Fixed point, Existence, Uniqueness, Boundary value problem.
\end{abstract}

\section{Introduction}

Boundary value problems for fractional differential equations with nonlocal boundary conditions constitute a very interesting and important class of problems (see $[4,5])$. Differential equations of fractional order with nonlocal boundary conditions arise in a variety of different areas of applied mathematics and physics. For example, heat conduction, chemical engineering, underground water flow, thermo-elasticity, and plasma physics can be reduced to nonlocal problems with integral boundary conditions. For more details, we refer the reader to $[6,25]$. Recently, by applying different fixed point theorems such as the Banach fixed point theorem, Schaefer's fixed point theorem, Krasnoselskii's fixed point theorem, the Leray-Schauder nonlinear alternative and the fixed point theorem of O'Regan, many researchers have obtained some interesting results of the existence and uniqueness of solutions to boundary value problems for fractional differential equations with nonlocal boundary value problems $[1,2,7,8,9,14,15,18,23,24]$ and the references therein. Ulam's stability problem [17] has been attracted by several famous researchers. Since then, a large number of monographs have been published in connection with

Received February 28, 2017; accepted March 14, 2019

2010 Mathematics Subject Classification. Primary 34A08; Secondary 05A30, 26D15 
various generalizations of Ulam's type stability theory or the Ulam-Hyers stability theory. For some recent development on Ulam's type stability, we refer the reader to $[3,12,16,17,19,20,21,22]$. The stability of fractional differential equations has been investigated by many authors $[19,21,22]$.

Motivated by the above papers, we study the existence, uniqueness and stability of solutions to the following fractional boundary value problem with tow Caputo fractional derivatives involving nonlocal boundary conditions:

$$
\left\{\begin{array}{l}
D^{\alpha}\left(D^{\beta}+\lambda\right) x(t)=f(t, x(t))+\int_{0}^{t} \frac{(t-s)^{\sigma-1}}{\Gamma(\sigma)} f(s, x(s)) d s, t \in[0, T], \\
x(0)=x_{0}+g(x), x(T)=\theta \int_{0}^{\eta} \frac{(\eta-s)^{p-1}}{\Gamma(p)} x(s) d s, 0<\eta<T
\end{array}\right.
$$

where $D^{\alpha}, D^{\beta}$ denote the Caputo fractional derivatives, with $0<\alpha, \beta \leq 1,1<$ $\alpha+\beta \leq 2, f:[0, T] \times \mathbb{R} \rightarrow \mathbb{R}$ and $g: C([0, T], \mathbb{R}) \rightarrow \mathbb{R}$ are given continuous functions, and $\sigma, p>0, \lambda, x_{0}, \theta$ are real constants In (1.1), $g(x)$ may be regarded as $g(x)=\sum_{j=0}^{m} k_{j} x\left(t_{j}\right)$, where $k_{j}, j=1, \ldots, m$ are given constants and $0<t_{0}<$ $\ldots<t_{m} \leq 1$.

The paper is organized as follows: In Section 2, we recall some preliminaries and lemmas that we need in the sequel. In Section 3, we present our main results for the existence, uniqueness and stability of solutions to the fractional boundary value problem (1.1). Some examples to illustrate our results are presented in Section 4.

\section{Preliminaries}

In this section, we present some useful definitions and lemmas [10, 11, 13]:

Definition 2.1. The Riemann-Liouville fractional integral operator of order $\vartheta \geq$ 0 , for a continuous function $f$ on $[a, b]$ is defined as:

$$
\begin{gathered}
I^{\vartheta} f(t)=\frac{1}{\Gamma(\vartheta)} \int_{a}^{t}(t-\tau)^{\vartheta-1} f(\tau) d \tau, \vartheta>0, a \leq t \leq b \\
I^{0} f(t)=f(t),
\end{gathered}
$$

where $\Gamma(\vartheta):=\int_{0}^{+\infty} e^{-u} u^{\vartheta-1} d u$.

Definition 2.2. The fractional derivative of $f \in C^{n}([a, b])$ in Caputo's sense is defined as:

$$
D^{\vartheta} f(t)=\frac{1}{\Gamma(n-\vartheta)} \int_{a}^{t}(t-\tau)^{n-\vartheta-1} f^{(n)}(\tau) d \tau, n-1<\vartheta, n \in N^{*}, a \leq t \leq b .
$$


The following lemmas give some properties of Riemann-Liouville fractional integrals and the Caputo fractional derivative $[10,11]$ :

Lemma 2.1. Let $\vartheta, s>0, f \in L^{1}([a, b])$. Then $I^{\vartheta} I^{s} f(t)=I^{\vartheta+s} f(t), D^{s} I^{s} f(t)=$ $f(t), t \in[a, b]$.

Lemma 2.2. Let $s>\vartheta>0, f \in L^{1}([a, b])$. Then $D^{\vartheta} I^{s} f(t)=I^{s-\vartheta} f(t), t \in[a, b]$.

We also give the following lemmas [10]:

Lemma 2.3. For $\vartheta>0$, the general solution to the fractional differential equation $D^{\vartheta} x(t)=0$ is given by

$$
x(t)=c_{0}+c_{1} t+c_{2} t^{2}+\ldots+c_{n-1} t^{n-1},
$$

where $c_{i} \in \mathbb{R}, i=0,1,2, . ., n-1, n=[\vartheta]+1$.

Lemma 2.4. Let $\vartheta>0$. Then

$$
I^{\vartheta} D^{\vartheta} x(t)=x(t)+c_{0}+c_{1} t+c_{2} t^{2}+\ldots+c_{n-1} t^{n-1},
$$

for some $c_{i} \in \mathbb{R}, i=0,1,2, \ldots, n-1, n=[\vartheta]+1$.

We also need the following auxiliary result:

Lemma 2.5. For a given $h \in C([0, T], \mathbb{R})$, the solution to the fractional boundary value problem

$$
\left\{\begin{array}{l}
D^{\alpha}\left(D^{\beta}+\lambda\right) x(t)=h(t), t \in[0, T], 0<\alpha, \beta \leq 1, \\
x(0)=x_{0}+g(x), x(T)=\theta I^{p} x(\eta),
\end{array}\right.
$$

is given by

$$
\begin{aligned}
(2.2) & x(t) \\
= & \int_{0}^{t} \frac{(t-s)^{\alpha+\beta-1}}{\Gamma(\alpha+\beta)} h(s) d s-\lambda \int_{0}^{t} \frac{(t-s)^{\beta-1}}{\Gamma(\beta)} x(s) d s \\
& -\frac{\Delta t^{\beta}}{\Gamma(\beta+1)} \int_{0}^{T} \frac{(T-s)^{\alpha+\beta-1}}{\Gamma(\alpha+\beta)} h(s) d s+\frac{\lambda \Delta t^{\beta}}{\Gamma(\beta+1)} \int_{0}^{T} \frac{(T-s)^{\beta-1}}{\Gamma(\beta)} x(s) d s \\
& +\frac{\Delta \theta t^{\beta}}{\Gamma(\beta+1)} \int_{0}^{\eta} \frac{(\eta-s)^{\alpha+\beta+p-1}}{\Gamma(\alpha+\beta+p)} h(s) d s-\frac{\lambda \Delta \theta t^{\beta}}{\Gamma(\beta+1)} \int_{0}^{\eta} \frac{(\eta-s)^{\beta+p-1}}{\Gamma(\beta+p)} x(s) d s \\
& +\left(\Delta \frac{\left(\theta \eta^{p}-\Gamma(p+1)\right) t^{\beta}}{\Gamma(p+1) \Gamma(\beta+1)}+1\right)\left(x_{0}+g(x)\right),
\end{aligned}
$$

where

$$
\Delta=\frac{\Gamma(\beta+p+1) \Gamma(\beta+1)}{\Gamma(\beta+p+1) T^{\beta}-\Gamma(\beta+1) \theta \eta^{\beta+p}}, \Gamma(\beta+p+1) T^{\beta} \neq \Gamma(\beta+1) \theta \eta^{\beta+p} .
$$


Proof. By Lemmas 5 and 6, we have

$$
x(t)=I^{\alpha+\beta} h(t)-\lambda I^{\beta} x(t)-\frac{c_{0}}{\Gamma(\beta+1)} t^{\beta}-c_{1},
$$

for some arbitrary constants $c_{0}, c_{1} \in \mathbb{R}$.

Using the boundary condition: $x(0)=x_{0}+g(x)$, we obtain

$$
c_{1}=-\left(x_{0}+g(x)\right) \text {. }
$$

Thanks to Lemma 3, we get

$$
I^{p} x(t)=I^{\alpha+\beta+p} h(t)-\lambda I^{\beta+p} x(t)-\frac{c_{0}}{\Gamma(\beta+p+1)} t^{p+\beta}-\frac{c_{1}}{\Gamma(p+1)} t^{p} .
$$

Applying the boundary condition: $x(T)=\theta I^{p} x(\eta)$, we obtain

$$
\begin{aligned}
c_{0}= & \Delta\left[I^{\alpha+\beta} h(T)-\lambda I^{\beta} x(T)-\theta I^{\alpha+\beta+p} h(\eta)+\lambda \theta I^{\beta+p} x(\eta)\right. \\
& \left.-\frac{\left(\theta \eta^{p}-\Gamma(p+1)\right)}{\Gamma(p+1)}\left(x_{0}+g(x)\right)\right],
\end{aligned}
$$

where $\Delta$ defined by (2.3). Substituting the value of $c_{0}$ and $c_{1}$ in (2.4), we obtain the solution (2.2).

In view of Lemma 4, we define the operator: $\phi: X \rightarrow X$ as

$$
\begin{aligned}
& (2.5) \phi x(t) \\
& =\int_{0}^{t} \frac{(t-s)^{\alpha+\beta-1}}{\Gamma(\alpha+\beta)} f(s, x(s)) d s-\lambda \int_{0}^{t} \frac{(t-s)^{\beta-1}}{\Gamma(\beta)} x(s) d s \\
& \quad-\frac{\Delta t^{\beta}}{\Gamma(\beta+1)} \int_{0}^{T} \frac{(T-s)^{\alpha+\beta-1}}{\Gamma(\alpha+\beta)} f(s, x(s)) d s+\frac{\lambda \Delta t^{\beta}}{\Gamma(\beta+1)} \int_{0}^{T} \frac{(T-s)^{\beta-1}}{\Gamma(\beta)} x(s) d s \\
& \quad+\frac{\Delta \theta t^{\beta}}{\Gamma(\beta+1)} \int_{0}^{\eta} \frac{(\eta-s)^{\alpha+\beta+p-1}}{\Gamma(\alpha+\beta+p)} f(s, x(s)) d s-\frac{\lambda \Delta \theta t^{\beta}}{\Gamma(\beta+1)} \int_{0}^{\eta} \frac{(\eta-s)^{\beta+p-1}}{\Gamma(\beta+p)} x(s) d s \\
& \quad+\left(\Delta \frac{\left(\theta \eta^{p}-\Gamma(p+1)\right) t^{\beta}}{\Gamma(p+1) \Gamma(\beta+1)}+1\right)\left(x_{0}+g(x)\right),
\end{aligned}
$$

We also introduce the operators $\phi_{1}, \phi_{2}: X \rightarrow X$, such that

$$
\begin{aligned}
& \left(2.6 \phi_{1} x(t)\right. \\
& =\int_{0}^{t} \frac{(t-s)^{\alpha+\beta-1}}{\Gamma(\alpha+\beta)} f(s, x(s)) d s-\lambda \int_{0}^{t} \frac{(t-s)^{\beta-1}}{\Gamma(\beta)} x(s) d s \\
& \quad-\frac{\Delta t^{\beta}}{\Gamma(\beta+1)} \int_{0}^{T} \frac{(T-s)^{\alpha+\beta-1}}{\Gamma(\alpha+\beta)} f(s, x(s)) d s+\frac{\lambda \Delta t^{\beta}}{\Gamma(\beta+1)} \int_{0}^{T} \frac{(T-s)^{\beta-1}}{\Gamma(\beta)} x(s) d s \\
& \quad+\frac{\Delta \theta t^{\beta}}{\Gamma(\beta+1)} \int_{0}^{\eta} \frac{(\eta-s)^{\alpha+\beta+p-1}}{\Gamma(\alpha+\beta+p)} f(s, x(s)) d s-\frac{\lambda \Delta \theta t^{\beta}}{\Gamma(\beta+1)} \int_{0}^{\eta} \frac{(\eta-s)^{\beta+p-1}}{\Gamma(\beta+p)} x(s) d s,
\end{aligned}
$$


and

$$
\phi_{2}(x)(t)=\left(\Delta \frac{\left(\theta \eta^{p}-\Gamma(p+1)\right) t^{\beta}}{\Gamma(p+1) \Gamma(\beta+1)}+1\right)\left(x_{0}+g(x)\right) .
$$

Clearly

$$
\phi(x)(t)=\phi_{1} x(t)+\phi_{2} x(t), t \in[0, T] .
$$

\section{Main Results}

We denote by $X=C([0, T], \mathbb{R})$ the Banach space of all continuous functions from $[0, T]$ into $\mathbb{R}$ endowed with a topology of uniform convergence with the norm defined by $\|x\|=\sup \{|x(t)|: t \in[0, T]\}$.

For computational convenience, we set the notations:

$$
\begin{aligned}
\Lambda= & \frac{T^{\alpha+\beta}}{\Gamma(\alpha+\beta+1)}+\frac{T^{\alpha+\beta+\sigma}}{\Gamma(\alpha+\beta+\sigma+1)} \\
& +\frac{|\Delta| T^{\beta}}{\Gamma(\beta+1)}\left[\frac{T^{\alpha+\beta}}{\Gamma(\alpha+\beta+1)}+\frac{T^{\alpha+\beta+\sigma}}{\Gamma(\alpha+\beta+\sigma+1)}\right. \\
& \left.+\frac{|\theta| \eta^{\alpha+\beta+p}}{\Gamma(\alpha+\beta+p+1)}+\frac{|\theta| \eta^{\alpha+\beta+p+\sigma}}{\Gamma(\alpha+\beta+p+\sigma+1)}\right] \\
& \Lambda_{1}=\frac{\left|\Delta\left(\theta \eta^{p}-\Gamma(p+1)\right)\right| T^{\beta}}{\Gamma(p+1) \Gamma(\beta+1)}+1 \\
\Lambda_{2}= & \frac{|\lambda| T^{\beta}}{\Gamma(\beta+1)}\left[1+\frac{|\Delta| T^{\beta}}{\Gamma(\beta+1)}+\frac{|\Delta \theta| \eta^{\beta+p}}{\Gamma(\beta+p+1)}\right]
\end{aligned}
$$

and

$$
\begin{aligned}
\rho= & {\left[\frac{T^{\alpha+\beta}}{\Gamma(\alpha+\beta+1)}+\frac{T^{\alpha+\beta+\sigma}}{\Gamma(\alpha+\beta+\sigma+1)}\right.} \\
& +\frac{|\Delta| T^{\beta}}{\Gamma(\beta+1)}\left(\frac{T^{\alpha+\beta}}{\Gamma(\alpha+\beta+1)}+\frac{T^{\alpha+\beta+\sigma}}{\Gamma(\alpha+\beta+\sigma+1)}\right. \\
& \left.\left.+\frac{|\theta| \eta^{\alpha+\beta+p}}{\Gamma(\alpha+\beta+p+1)}+\frac{|\theta| \eta^{\alpha+\beta+p+\sigma}}{\Gamma(\alpha+\beta+p+\sigma+1)}\right)\right]\|\gamma\| .
\end{aligned}
$$

Now, we impose the following hypotheses:

$(H 1)$ : There exists a constant $\omega>0$ such that for all $t \in[0, T]$ and $x, y \in$ $C([0, T], \mathbb{R})$, we have $|f(t, x)-f(t, y)| \leq \omega\|x-y\|$, 
(H2) : There exists a positive constant $\varpi<\frac{1}{\Lambda_{1}}$ and a continuous function $\varphi:[0, \infty) \rightarrow[0, \infty)$ such that $\varphi(u) \leq \varpi u$ and $|g(x)-g(y)| \leq \varpi(\|x-y\|)$, for all $x, y \in C([0, T])$.

$(H 3): g(0)=0$.

$(H 4)$ : There exists a non-negative function $\gamma(t) \in C([0, T], \mathbb{R})$ and there exists a nondecreasing function $\psi:[0, \infty) \rightarrow(0, \infty)$, such that $|f(t, x)| \leq \gamma(t) \psi(|x|)$ for all $(t, x) \in[0, T] \times X$.

$(H 5): \sup _{r \in(0, \infty)} \frac{r}{\rho \psi(r)+\Lambda_{1}\left|x_{0}\right|}>\frac{1}{1-\left(\Lambda_{2}+\Lambda_{1} \varpi\right)}$, where $\Lambda_{1}, \rho$ and $\Lambda_{2}$ are given respectively in (3.2), (3.3) and (3.4).

\subsection{Existence and uniqueness of solutions}

The first result is concerned with the existence and uniqueness of solutions to fractional boundary value problems and is based on the Banach contraction principle.

Theorem 3.1. Let $f:[0, T] \times \mathbb{R} \rightarrow \mathbb{R}$ be a continuous function. Assume that (H1) and (H2) hold. If the inequality

$$
\Lambda \omega+\Lambda_{1} \varpi<1-\Lambda_{2},
$$

is valid, then the fractional boundary value problem (1.1) has a unique solution on $[0, T]$.

Proof. For $x, y \in X$ and by $(H 1)$ and $(H 2)$ we have:

$$
\begin{aligned}
& \|\phi(x)-\phi(y)\| \\
\leq & \sup _{t \in[0, T]}\left\{\int_{0}^{t} \frac{(t-s)^{\alpha+\beta-1}}{\Gamma(\alpha+\beta)}|f(s, x(s))-f(s, y(s))| d s\right. \\
& +\int_{0}^{t} \frac{(t-s)^{\alpha+\beta+\sigma-1}}{\Gamma(\alpha+\beta+\sigma)}|f(s, x(s))-f(s, y(s))| d s \\
& +|\lambda| \int_{0}^{t} \frac{(t-s)^{\beta-1}}{\Gamma(\beta)}|x(s)-y(s)| d s \\
& +\frac{|\Delta| t^{\beta}}{\Gamma(\beta+1)} \int_{0}^{T} \frac{(T-s)^{\alpha+\beta-1}}{\Gamma(\alpha+\beta)}|f(s, x(s))-f(s, y(s))| d s \\
& +\frac{|\Delta| t^{\beta}}{\Gamma(\beta+1)} \int_{0}^{T} \frac{(T-s)^{\alpha+\beta+\sigma-1}}{\Gamma(\alpha+\beta+\sigma)}|f(s, x(s))-f(s, y(s))| d s \\
& +\frac{|\lambda||\Delta| t^{\beta}}{\Gamma(\beta+1)} \int_{0}^{T} \frac{(T-s)^{\beta-1}}{\Gamma(\beta)}|x(s)-y(s)| d s \\
& +\frac{|\Delta \|||\theta| t^{\beta}}{\Gamma(\beta+1)} \int_{0}^{\eta} \frac{(\eta-s)^{\alpha+\beta+p-1}}{\Gamma(\alpha+\beta+p)}|f(s, x(s))-f(s, y(s))| d s
\end{aligned}
$$




$$
\begin{aligned}
& +\frac{|\Delta||\theta| t^{\beta}}{\Gamma(\beta+1)} \int_{0}^{\eta} \frac{(\eta-s)^{\alpha+\beta+p+\sigma-1}}{\Gamma(\alpha+\beta+p+\sigma)}|f(s, x(s))-f(s, y(s))| d s \\
& +\frac{|\Delta||\theta| t^{\beta}}{\Gamma(\beta+1)} \int_{0}^{\eta} \frac{(\eta-s)^{\beta+p-1}}{\Gamma(\beta+p)}|x(s)-y(s)| d s \\
& \left.+\left(\frac{\left|\Delta\left(\theta \eta^{p}-\Gamma(p+1)\right)\right| T^{\beta}}{\Gamma(p+1) \Gamma(\beta+1)}+1\right)|g(x)-g(y)|\right\} \\
& \leq \sup _{t \in[0, T]}\left\{\left[\int_{0}^{t} \frac{(t-s)^{\alpha+\beta-1}}{\Gamma(\alpha+\beta)} d s+\int_{0}^{t} \frac{(t-s)^{\alpha+\beta+\sigma-1}}{\Gamma(\alpha+\beta+\sigma)} d s\right.\right. \\
& +\frac{|\Delta| t^{\beta}}{\Gamma(\beta+1)} \int_{0}^{T} \frac{(T-s)^{\alpha+\beta-1}}{\Gamma(\alpha+\beta)} d s+\frac{|\Delta| t^{\beta}}{\Gamma(\beta+1)} \int_{0}^{T} \frac{(T-s)^{\alpha+\beta+\sigma-1}}{\Gamma(\alpha+\beta+\sigma)} d s \\
& \left.+\frac{|\Delta \theta| t^{\beta}}{\Gamma(\beta+1)} \int_{0}^{\eta} \frac{(\eta-s)^{\alpha+\beta+p-1}}{\Gamma(\alpha+\beta+p)} d s+\frac{|\Delta \theta| t^{\beta}}{\Gamma(\beta+1)} \int_{0}^{\eta} \frac{(\eta-s)^{\alpha+\beta+p+\sigma-1}}{\Gamma(\alpha+\beta+p+\sigma)} d s\right] \omega \\
& +|\lambda|\left[\int_{0}^{t} \frac{(t-s)^{\beta-1}}{\Gamma(\beta)} d s+\frac{|\Delta| t^{\beta}}{\Gamma(\beta+1)} \int_{0}^{T} \frac{(T-s)^{\beta-1}}{\Gamma(\beta)} d s\right. \\
& \left.\left.+\frac{|\Delta \theta| t^{\beta}}{\Gamma(\beta+1)} \int_{0}^{\eta} \frac{(\eta-s)^{\beta+p-1}}{\Gamma(\beta+p)} d s\right]+\left(\frac{\left|\Delta\left(\theta \eta^{p}-\Gamma(p+1)\right)\right| t^{\beta}}{\Gamma(p+1) \Gamma(\beta+1)}+1\right) \varpi\right\}\|x-y\| \\
& \leq\left\{\left[\frac{T^{\alpha+\beta}}{\Gamma(\alpha+\beta+1)}+\frac{T^{\alpha+\beta+\sigma}}{\Gamma(\alpha+\beta+\sigma+1)}+\frac{|\Delta| T^{\beta}}{\Gamma(\beta+1)}\left(\frac{T^{\alpha+\beta}}{\Gamma(\alpha+\beta+1)}\right.\right.\right. \\
& \left.\left.+\frac{T^{\alpha+\beta+\sigma}}{\Gamma(\alpha+\beta+\sigma+1)}+\frac{|\theta| \eta^{\alpha+\beta+p}}{\Gamma(\alpha+\beta+p+1)}+\frac{|\theta| \eta^{\alpha+\beta+p+\sigma}}{\Gamma(\alpha+\beta+p+\sigma+1)}\right)\right] \omega \\
& \left.+\left[\frac{|\lambda| T^{\beta}}{\Gamma(\beta+1)}\left(1+\frac{|\Delta| T^{\beta}}{\Gamma(\beta+1)}+\frac{|\Delta \theta| \eta^{\beta+p}}{\Gamma(\beta+p+1)}\right)\right]+\left(\frac{\left|\Delta\left(\theta \eta^{p}-\Gamma(p+1)\right)\right| T^{\beta}}{\Gamma(p+1) \Gamma(\beta+1)}+1\right) \varpi\right\}\|x-y\| \\
& =\left(\Lambda \omega+\Lambda_{2}+\Lambda_{1} \varpi\right)\|x-y\| \text {. }
\end{aligned}
$$

Thanks to (3.5), we conclude that $\phi$ is a contraction. As a consequence of the Banach fixed point theorem, we deduce that $\phi$ has a fixed point which is a solution to the fractional boundary value problem (1.1).

In the next result, we prove the existence of solutions to the fractional boundary value problem by applying the following Lemma.

Lemma 3.1. (O'Regan Lemma) [15]. Denote by $V$ an open set in a closed, convex set $C$ of a Banach space $E$. Assume $0 \in V$. Also assume that $\phi(\bar{V})$ is bounded and that $\phi: \bar{V} \rightarrow C$ is given by $\phi=\phi_{1}+\phi_{2}$, in which $\phi_{1}: \bar{V} \rightarrow E$ is continuous and completely continuous and $\phi_{2}: \bar{V} \rightarrow E$ is a nonlinear contraction (i.e., there exists a nonnegative nondecreasing function $\varphi:(0, \infty) \rightarrow(0, \infty)$ satisfying $\varphi(u)<u$ for $v>0$ such that $\left\|\phi_{2}(x)-\phi_{2}(y)\right\| \leq \varphi\|x-y\|$ for all $\left.x, y \in \bar{V}\right)$. Then, either

$(I): \phi$ has a fixed point $x \in \bar{V}$; or

$(I I)$ : there exists a point $x \in \partial V$ and $0<\mu<1$ with $x=\mu \phi(x)$, where $\bar{V}$ (respectively $\partial V$ ) represents the closure (respectively the boundary) of $V$. 
Let

$$
\Omega:=\{x \in C([0, T], \mathbb{R}):\|x\|<\delta\},
$$

and denote the maximum number by

$$
N_{\delta}:=\max \{|f(t, x)|:(t, x) \in[0, T] \times[\delta,-\delta]\} .
$$

Theorem 3.2. Let $f:[0, T] \times \mathbb{R} \rightarrow \mathbb{R}$ be a continuous function. Suppose that $(H 2),(H 3),(H 4)$ and $(H 5)$ are satisfied.

Then the boundary value problem (1.1) has at least one solution on $[0, T]$.

Proof. Consider the operator $\phi: X \rightarrow X$ defined by:

$$
\phi(x)(t):=\phi_{1}(x)(t)+\phi_{2}(x)(t), t \in[0, T],
$$

where the operators $\phi_{1}$ and $\phi_{2}$ are defined respectively in (2.6) and (2.7).

From $\left(H_{5}\right)$ there exists a number $\delta_{0}>0$ such that

$$
\frac{\delta_{0}}{\rho \psi\left(\delta_{0}\right)+\Lambda_{1}\left|x_{0}\right|}>\frac{1}{1-\left(\Lambda_{2}+\Lambda_{1} \varpi\right)} .
$$

We shall prove that the operators $\phi_{1}$ and $\phi_{2}$ satisfy all the conditions in Lemma 9.

Step 1 : We show that the operator $\phi_{1}: \bar{\Omega}_{\delta_{0}} \rightarrow X$ is continuous and completely continuous. Let us consider the set

$$
\bar{\Omega}_{\delta_{0}}:=\left\{x \in C([0, T], \mathbb{R}):\|x\| \leq \delta_{0}\right\},
$$

and show that $\phi_{1}\left(\bar{\Omega}_{\delta_{0}}\right)$ is bounded. For each $x \in \bar{\Omega}_{\delta_{0}}$, we have

$$
\begin{gathered}
\leq \sup _{t \in[0, T]}\left\{\int_{0}^{t} \frac{(t-s)^{\alpha+\beta-1}}{\Gamma(\alpha+\beta)}|f(s, x(s))| d s+\int_{0}^{t} \frac{(t-s)^{\alpha+\beta+\sigma-1}}{\Gamma(\alpha+\beta+\sigma)}|f(s, x(s))| d s\right. \\
+|\lambda| \int_{0}^{t} \frac{(t-s)^{\beta-1}}{\Gamma(\beta)}|x(s)| d s+\frac{|\Delta| t^{\beta}}{\Gamma(\beta+1)}\left[\int_{0}^{T} \frac{(T-s)^{\alpha+\beta-1}}{\Gamma(\alpha+\beta)}|f(s, x(s))| d s\right. \\
+\int_{0}^{T} \frac{(T-s)^{\alpha+\beta+\sigma-1}}{\Gamma(\alpha+\beta+\sigma)}|f(s, x(s))| d s+|\lambda| \int_{0}^{T} \frac{(T-s)^{\beta-1}}{\Gamma(\beta)}|x(s)| d s \\
+|\theta| \int_{0}^{\eta} \frac{(\eta-s)^{\alpha+\beta+p-1}}{\Gamma(\alpha+\beta+p)}|f(s, x(s))| d s+|\theta| \int_{0}^{\eta} \frac{(\eta-s)^{\alpha+\beta+p+\sigma-1}}{\Gamma(\alpha+\beta+p+\sigma)}|f(s, x(s))| d s \\
\left.\left.+|\lambda \theta| \int_{0}^{\eta} \frac{(\eta-s)^{\beta+p-1}}{\Gamma(\beta+p)}|x(s)| d s\right]\right\} \\
\leq \quad\left[\frac{T^{\alpha+\beta}}{\Gamma(\alpha+\beta+1)}+\frac{T^{\alpha+\beta+\sigma}}{\Gamma(\alpha+\beta+\sigma+1)}+\frac{|\Delta| T^{\beta}}{\Gamma(\beta+1)}\left(\frac{T^{\alpha+\beta}}{\Gamma(\alpha+\beta+1)}\right.\right. \\
\left.\left.\quad+\frac{T^{\alpha+\beta+\sigma}}{\Gamma(\alpha+\beta+\sigma+1)}+\frac{|\theta| \eta^{\alpha+\beta+p}}{\Gamma(\alpha+\beta+p+1)}+\frac{|\theta| \eta^{\alpha+\beta+p+\sigma}}{\Gamma(\alpha+\beta+p+\sigma+1)}\right)\right] N_{\delta_{0}}\|\gamma\| \\
\quad+\frac{|\lambda| T^{\beta}}{\Gamma(\beta+1)}\left(1+\frac{|\Delta| T^{\beta}}{\Gamma(\beta+1)}+\frac{|\Delta \theta| \eta^{\beta+p}}{\Gamma(\beta+p+1)}\right) \delta_{0} \\
=\quad \Lambda \delta_{\delta_{0}}\|\gamma\|+\Lambda_{2} \delta_{0} .
\end{gathered}
$$


Thus the operator $\phi_{1}\left(\bar{\Omega}_{\delta_{0}}\right)$ is uniformly bounded. For any $0 \leq t_{1}<t_{2} \leq T$, we have

$$
\begin{aligned}
&\left|\phi_{1} x\left(t_{2}\right)-\phi_{1} x\left(t_{1}\right)\right| \\
& \leq \int_{0}^{t_{1}} \frac{\left.\left[t_{2}-s\right)^{\alpha+\beta-1}-\left(t_{1}-s\right)^{\alpha+\beta-1}\right]}{\Gamma(\alpha+\beta)}|f(s, x(s))| d s+\int_{t_{1}}^{t_{2}} \frac{\left(t_{2}-s\right)^{\alpha+\beta-1}}{\Gamma(\alpha+\beta)}|f(s, x(s))| d s \\
&+ \int_{0}^{t_{1}} \frac{\left[\left(t_{2}-s\right)^{\alpha+\beta+\sigma-1}-\left(t_{1}-s\right)^{\alpha+\beta+\sigma-1}\right]}{\Gamma(\alpha+\beta+\sigma)}|f(s, x(s))| d s+\int_{t_{1}}^{t_{2}} \frac{\left(t_{2}-s\right)^{\alpha+\beta+\sigma-1}}{\Gamma(\alpha+\beta+\sigma)}|f(s, x(s))| d s \\
&+|\lambda| \int_{0}^{t_{1}} \frac{\left[\left(t_{2}-s\right)^{\beta-1}-\left(t_{1}-s\right)^{\beta-1}\right]}{\Gamma(\beta)}|x(s)| d s+|\lambda| \int_{t_{1}}^{t_{2}} \frac{\left(t_{2}-s\right)^{\beta-1}}{\Gamma(\beta)}|x(s)| d s \\
&+\mid \frac{\left|t_{2}^{\beta}-t_{1}^{\beta}\right|}{\Gamma(\beta+1)}\left[|\Delta| \int_{0}^{T} \frac{(T-s)^{\alpha+\beta-1}}{\Gamma(\alpha+\beta)}|f(s, x(s))| d s+|\Delta| \int_{0}^{T} \frac{(T-s)^{\alpha+\beta+\sigma-1}}{\Gamma(\alpha+\beta+\sigma)}|f(s, x(s))| d s\right. \\
&+|\lambda \Delta| \int_{0}^{T} \frac{(T-s)^{\beta-1}}{\Gamma(\beta)}|x(s)| d s+|\Delta \theta| \int_{0}^{\eta} \frac{(\eta-s)^{\alpha+\beta+p-1}}{\Gamma(\alpha+\beta+p)}|f(s, x(s))| d s \\
&\left.+|\Delta \theta| \int_{0}^{\eta} \frac{(\eta-s)^{\alpha+\beta+p+\sigma-1}}{\Gamma(\alpha+\beta+p+\sigma)}|f(s, x(s))| d s+|\lambda \Delta \theta| \int_{0}^{\eta} \frac{(\eta-s)^{\beta+p-1}}{\Gamma(\beta+p)}|x(s)| d s\right] \\
& \leq \quad \frac{N_{\delta_{0}}\|\gamma\|}{\Gamma(\alpha+\beta+1)}\left|t_{2}^{\alpha+\beta}-t_{1}^{\alpha+\beta}\right|+\frac{N_{\delta_{0}}\|\gamma\|}{\Gamma(\alpha+\beta+\sigma+1)}\left|t_{2}^{\alpha+\beta+\sigma}-t_{1}^{\alpha+\beta+\sigma}\right| \\
&+\left[\frac { | \Delta | N _ { \delta _ { 0 } } \| \gamma \| } { \Gamma ( \beta + 1 ) } \left(\frac{T^{\alpha+\beta}}{\Gamma(\alpha+\beta+1)}+\frac{T^{\alpha+\beta+\sigma}}{\Gamma(\alpha+\beta+\sigma+1)}\right.\right. \\
&\left.+\frac{|\theta| \eta^{\alpha+\beta+p}}{\Gamma(\alpha+\beta+p+1)}+\frac{|\theta| \eta^{\alpha+\beta+p+\sigma}}{\Gamma(\alpha+\beta+p+\sigma+1)}\right) \\
&\left.+\frac{|\lambda| \delta_{0}}{\Gamma(\beta+1)}\left(1+\frac{|\Delta| T^{\beta}}{\Gamma(\beta+1)}+\frac{|\Delta \theta| \eta^{\beta+p}}{\Gamma(\beta+p+1)}\right)\right]\left|t_{2}^{\beta}-t_{1}^{\beta}\right|
\end{aligned}
$$

which is independent of $x$ and tends to zero as $t_{2} \rightarrow t_{1}$. Thus, $\phi_{1}$ is equicontinuous. Hence, by the Arzelà-Ascoli theorem, $\phi_{1}\left(\bar{\Omega}_{\delta_{0}}\right)$ is a relatively compact set. Now, let the sequence $x_{n} \subset \bar{\Omega}_{\delta_{0}}$ with $x_{n} \rightarrow x$. Then $x_{n}(t) \rightarrow x(t)$ uniformly valid on $[0, T]$, then for each $t \in[0, T]$, we have. From the uniform continuity of $f(t, x)$ on the compact set $[0, T] \times\left[\delta_{0},-\delta_{0}\right]$, it follows that $\left\|f\left(t, x_{n}(t)\right)-f(t, x(t))\right\| \rightarrow 0$ is uniformly valid on $J$. Hence $\left\|\phi_{1}\left(x_{n}\right)(t)-\phi_{1}(x)(t)\right\| \rightarrow 0$ as $n \rightarrow \infty$, which proves the continuity of $\phi_{1}\left(\bar{\Omega}_{\delta_{0}}\right)$.

Step $2:$ The operator $\phi_{2}: \bar{\Omega}_{\delta_{0}} \rightarrow X$ is contractive, this is the consequence of $\left(H_{2}\right)$.

Step3 : The set $\phi_{2}\left(\bar{\Omega}_{\delta_{0}}\right)$ is bounded. For any $x \in \bar{\Omega}_{\delta_{0}}$ and by $\left(H_{2}\right)$ and $\left(H_{3}\right)$, we have

$$
\left\|\phi_{2}(x)\right\| \leq \Lambda_{1}\left(\left|x_{0}\right|+\varpi \delta_{0}\right),
$$

combining, with the set $\phi_{1}\left(\bar{\Omega}_{\delta_{0}}\right)$ being bounded, then the set $\phi\left(\bar{\Omega}_{\delta_{0}}\right)$ is bounded. Step 4 : Finally, will be show that the case $(I I)$ in Lemma 9 does not hold. On the contrary, we suppose that $(I I)$ holds. Then, there exist $\mu \in(0,1)$ and $x \in \partial \Omega_{\delta_{0}}$, 
such that $x=\mu \phi(x)$. So we have $\|x\|=\delta_{0}$ and

$$
\begin{aligned}
x & (t) \\
= & \mu\left[\int_{0}^{t} \frac{(t-s)^{\alpha+\beta-1}}{\Gamma(\alpha+\beta)} f(s, x(s)) d s+\int_{0}^{t} \frac{(t-s)^{\alpha+\beta+\sigma-1}}{\Gamma(\alpha+\beta+\sigma)} f(s, x(s)) d s\right. \\
& -\lambda \int_{0}^{t} \frac{(t-s)^{\beta-1}}{\Gamma(\beta)} x(s) d s-\frac{\Delta t^{\beta}}{\Gamma(\beta+1)} \int_{0}^{T} \frac{(T-s)^{\alpha+\beta-1}}{\Gamma(\alpha+\beta)} f(s, x(s)) d s \\
& -\frac{\Delta t^{\beta}}{\Gamma(\beta+1)} \int_{0}^{T} \frac{(T-s)^{\alpha+\beta+\sigma-1}}{\Gamma(\alpha+\beta+\sigma)} f(s, x(s)) d s+\frac{\lambda \Delta t^{\beta}}{\Gamma(\beta+1)} \int_{0}^{T} \frac{(T-s)^{\beta-1}}{\Gamma(\beta)} x(s) d s \\
& +\frac{\Delta \theta t^{\beta}}{\Gamma(\beta+1)} \int_{0}^{\eta} \frac{(\eta-s)^{\alpha+\beta+p-1}}{\Gamma(\alpha+\beta+p)} f(s, x(s)) d s+\frac{\Delta \theta t^{\beta}}{\Gamma(\beta+1)} \int_{0}^{\eta} \frac{(\eta-s)^{\alpha+\beta+p+\sigma-1}}{\Gamma(\alpha+\beta+p+\sigma)} f(s, x(s)) d s \\
& \left.-\frac{\lambda \Delta \theta t^{\beta}}{\Gamma(\beta+1)} \int_{0}^{\eta} \frac{(\eta-s)^{\beta+p-1}}{\Gamma(\beta+p)} x(s) d s+\left(\frac{\Delta\left(\theta \eta^{p}-\Gamma(p+1)\right) t^{\beta}}{\Gamma(p+1) \Gamma(\beta+1)}+1\right)\left(x_{0}+g(x)\right)\right], t \in[0, T] .
\end{aligned}
$$

Using the hypotheses $(H 3)-(H 5)$ we get

$$
\begin{aligned}
\|x\| \leq & {\left[\int_{0}^{t} \frac{(t-s)^{\alpha+\beta-1}}{\Gamma(\alpha+\beta)} \gamma(s) d s+\int_{0}^{t} \frac{(t-s)^{\alpha+\beta+\sigma-1}}{\Gamma(\alpha+\beta+\sigma)} \gamma(s) d s\right.} \\
& +\frac{|\Delta| t^{\beta}}{\Gamma(\beta+1)} \int_{0}^{T} \frac{(T-s)^{\alpha+\beta-1}}{\Gamma(\alpha+\beta)} \gamma(s) d s \\
& +\frac{|\Delta| t^{\beta}}{\Gamma(\beta+1)} \int_{0}^{T} \frac{(T-s)^{\alpha+\beta+\sigma-1}}{\Gamma(\alpha+\beta+\sigma)} \gamma(s) d s \\
& +\frac{|\Delta \theta| t^{\beta}}{\Gamma(\beta+1)} \int_{0}^{\eta} \frac{(\eta-s)^{\alpha+\beta+p-1}}{\Gamma(\alpha+\beta+p)} \gamma(s) d s \\
& \left.+\frac{|\Delta \theta| t^{\beta}}{\Gamma(\beta+1)} \int_{0}^{\eta} \frac{(\eta-s)^{\alpha+\beta+p+\sigma-1}}{\Gamma(\alpha+\beta+p+\sigma)} \gamma(s) d s\right] \psi(\|x\|) \\
& +|\lambda|\left[\int_{0}^{t} \frac{(t-s)^{\beta-1}}{\Gamma(\beta)} d s+\frac{|\Delta| t^{\beta}}{\Gamma(\beta+1)} \int_{0}^{T} \frac{(T-s)^{\beta-1}}{\Gamma(\beta)} d s\right. \\
& \left.\frac{|\Delta \theta| t^{\beta}}{\Gamma(\beta+1)} \int_{0}^{\eta} \frac{(\eta-s)^{\beta+p-1}}{\Gamma(\beta+p)} d s\right]\|x\| \\
& +\left(\frac{\left|\Delta\left(\theta \eta^{p}-\Gamma(p+1)\right)\right| t^{\beta}}{\Gamma(p+1) \Gamma(\beta+1)}+1\right)\left(x_{0}+\varpi\|x\|\right) .
\end{aligned}
$$

By (3.3) and (3.7), we obtain

$$
\begin{aligned}
\delta_{0} \leq & {\left[\frac{T^{\alpha+\beta}}{\Gamma(\alpha+\beta+1)}+\frac{T^{\alpha+\beta+\sigma}}{\Gamma(\alpha+\beta+\sigma+1)}+\frac{|\Delta| T^{\beta}}{\Gamma(\beta+1)}\left(\frac{T^{\alpha+\beta}}{\Gamma(\alpha+\beta+1)}+\frac{T^{\alpha+\beta+\sigma}}{\Gamma(\alpha+\beta+\sigma+1)}\right.\right.} \\
& \left.\left.+\frac{|\theta| \eta^{\alpha+\beta+p}}{\Gamma(\alpha+\beta+p+1)}+\frac{|\theta| \eta^{\alpha+\beta+p+\sigma}}{\Gamma(\alpha+\beta+p+\sigma+1)}\right)\right]\|\rho\| \psi\left(\delta_{0}\right) \\
& +\left[\frac{|\lambda| T^{\beta}}{\Gamma(\beta+1)}\left(1+\frac{|\Delta| T^{\beta}}{\Gamma(\beta+1)}+\frac{|\Delta \theta| \eta^{\beta+p}}{\Gamma(\beta+p+1)}\right)+\left(\frac{\left|\Delta\left(\theta \eta^{p}-\Gamma(p+1)\right)\right| T^{\beta}}{\Gamma(p+1) \Gamma(\beta+1)}+1\right) \varpi\right] \delta_{0} \\
& +\left(\frac{\left|\Delta\left(\theta \eta^{p}-\Gamma(p+1)\right)\right| T^{\beta}}{\Gamma(p+1) \Gamma(\beta+1)}+1\right)\left|x_{0}\right|,
\end{aligned}
$$


which implies

$$
\delta_{0} \leq \rho \psi\left(\delta_{0}\right)+\left(\Lambda_{2}+\Lambda_{1} \varpi\right) \delta_{0}+\Lambda_{1}\left|x_{0}\right| .
$$

However,

$$
\frac{\delta_{0}}{\rho \psi\left(\delta_{0}\right)+\Lambda_{1}\left|x_{0}\right|} \leq \frac{1}{1-\left(\Lambda_{2}+\Lambda_{1} \varpi\right)}
$$

which contradicts (3.6). Consequently, the operators $\phi_{1}$ and $\phi_{2}$ satisfy all the conditions in Lemma 9. Hence, the operator $\phi$ has at least one fixed point $x \in \bar{\Omega}_{\delta_{0}}$, which is the solution of the fractional boundary value problem (1.1). This completes the proof.

\subsection{Ulam-Hyers stability}

In this section, we will study Ulam's type stability of the fractional boundary value problem (1.1).

Let $\varepsilon>0$, we consider the equation

$$
D^{\alpha}\left(D^{\beta}+\lambda\right) x(t)=f(t, x(t))+\int_{0}^{t} \frac{(t-s)^{\sigma-1}}{\Gamma(\sigma)} f(s, x(s)) d s
$$

and the following inequality

$$
\left|D^{\alpha}\left(D^{\beta}+\lambda\right) y(t)-f(t, y(t))-\int_{0}^{t} \frac{(t-s)^{\sigma-1}}{\Gamma(\sigma)} f(s, y(s)) d s\right| \leq \varepsilon, t \in[0, T],
$$

with $y(0)=y_{0}+g(y), y(T)=\theta I^{p} y(\eta)$.

Definition 3.1. The fractional boundary value problem (1.1) is Ulam-Hyers stable if there exists a real number $k>0$ such that for each solution $y \in X$ to the inequality (3.8) there exists a solution $x \in X$ of the fractional boundary value problem (1.1) with

$$
\|x-y\| \leq k \varepsilon .
$$

Definition 3.2. The fractional boundary value problem (1.1) is generalized UlamHyers stable if there exists $z \in C\left(\mathbb{R}^{+}, \mathbb{R}^{+}\right), z(0)=0$ such that for each solution $y \in X$ to the inequality (3.8), there exists a solution $x \in X$ of the fractional boundary value problem (1.1) with

$$
\|x-y\| \leq z(\varepsilon) .
$$

Theorem 3.3. Let $f:[0, T] \times \mathbb{R} \rightarrow \mathbb{R}$ be a continuous function. Suppose that $\left(H_{1}\right)-\left(H_{4}\right)$ holds. In addition, we assume that:

$$
\begin{gathered}
\text { If } \left.H_{6}\right) \sup _{t \in[0, T]}\left|D^{\alpha}\left(D^{\beta}+\lambda\right) x(t)\right| \geq \Lambda N_{\delta_{0}}\|\gamma\|+\left(\Lambda_{2}+\Lambda_{1} \varpi\right) \delta_{0}+\Lambda_{1}\left|x_{0}\right| . \\
\text { 9) } \\
\omega<\frac{\Gamma(\sigma+1)}{\left[\Gamma(\sigma+1)+T^{\sigma}\right]},
\end{gathered}
$$

then the fractional boundary value problem (1.1) has the Ulam-Hyers stability in X. 
Proof. For each $\varepsilon>0, y \in X$, we have

$$
\left|D^{\alpha}\left(D^{\beta}+\lambda\right) y(t)-f(t, y(t))-\int_{0}^{t} \frac{(t-s)^{\sigma-1}}{\Gamma(\sigma)} f(s, y(s)) d s\right| \leq \varepsilon,
$$

with $y(0)=y_{0}+g(y), y(T)=\theta I^{p} y(\eta)$.

Let us denote by $x \in X$ the unique solution of the fractional boundary value problem(1.1).

According to the assumptions of Theorem 8, we have

$$
|x(t)| \leq \Lambda N_{\delta_{0}}\|\gamma\|+\left(\Lambda_{2}+\Lambda_{1} \varpi\right) \delta_{0}+\Lambda_{1}\left|x_{0}\right|, t \in[0, T] .
$$

By $\left(H_{6}\right)$, we get

$$
\sup _{t \in[0, T]}|x(t)| \leq \sup _{t \in[0, T]}\left|D^{\alpha}\left(D^{\beta}+\lambda\right) x(t)\right| .
$$

Then

$$
\begin{aligned}
\sup _{t \in[0, T]}|x(t)-y(t)| \leq \sup _{t \in[0, T]}\left|D^{\alpha}\left(D^{\beta}+\lambda\right)(x(t)-y(t))\right| \\
\leq \sup _{t \in[0, T]} \mid D^{\alpha}\left(D^{\beta}+\lambda\right) x(t)-f(t, x(t))-\int_{0}^{t} \frac{(t-s)^{\sigma-1}}{\Gamma(\sigma)} f(s, x(s)) d s \\
\quad-D^{\alpha}\left(D^{\beta}+\lambda\right) y(t)+f(t, y(t))+\int_{0}^{t} \frac{(t-s)^{\sigma-1}}{\Gamma(\sigma)} f(s, y(s)) d s \\
+f(t, x(t))+\int_{0}^{t} \frac{(t-s)^{\sigma-1}}{\Gamma(\sigma)} f(s, x(s)) d s \\
\quad-f(t, y(t))-\int_{0}^{t} \frac{(t-s)^{\sigma-1}}{\Gamma(\sigma)} f(s, y(s)) d s \mid \\
\leq \quad 2 \varepsilon+\left(1+\frac{T^{\sigma}}{\Gamma(\sigma+1)}\right) \omega \sup _{t \in[0, T]}|x(t)-y(t)| .
\end{aligned}
$$

Hence

$$
\|x-y\| \leq \frac{2}{1-\left(1+\frac{T^{\sigma}}{\Gamma(\sigma+1)}\right) \omega} \varepsilon=k \varepsilon .
$$

Thus, the fractional boundary value problem (1.1) has the Ulam-Hyers stability in $X$.

Remark 3.1. By putting $z(\varepsilon)=k \varepsilon, z(\varepsilon)=0$ yields that the fractional boundary value problem (1.1) has the generalized Ulam-Hyers stability in $X$.

\section{Examples}

To illustrate our main results, we treat the following examples. 
Example 4.1. Let us consider the following fractional boundary value problem:

$$
\left\{\begin{array}{l}
D^{\frac{1}{2}}\left(D^{\frac{1}{2}}+\frac{3}{20}\right) x(t)=\left(\frac{e^{-\pi t}|x(t)|}{\left(25 \sqrt{\pi}+e^{-\pi t}\right)(1+|x(t)|)}+\frac{1}{2}+\cosh \left(t^{2}+2\right)\right) \\
+\int_{0}^{t} \frac{(t-s)^{\frac{3}{2}}}{\Gamma\left(\frac{5}{2}\right)}\left(\frac{e^{-\pi s}|x(s)|}{\left(25 \sqrt{\pi}+e^{-\pi s}\right)(1+|x(s)|)}+\frac{1}{2}+\cosh \left(s^{2}+2\right)\right) d s, t \in[0,1], \\
x(0)=\frac{\sqrt{2}}{3}+\frac{1}{19} x(\zeta), x(1)=\frac{5}{6} \int_{0}^{\frac{1}{4}} \frac{\left(\frac{1}{4}-s\right)^{\frac{1}{3}}}{\Gamma\left(\frac{4}{3}\right)} d s, 0<\zeta<1 .
\end{array}\right.
$$

with $\alpha=\frac{1}{2}, \beta=\frac{1}{2}, \lambda=\frac{3}{20}, \sigma=\frac{5}{2}, \theta=\frac{5}{6}, p=\frac{4}{3}, \eta=\frac{1}{4}$ and

$$
f(t, x)=\frac{e^{-\pi t}|x(t)|}{\left(25 \sqrt{\pi}+e^{-\pi t}\right)(1+|x(t)|)}+\frac{1}{2}+\cosh \left(t^{2}+2\right), g(x)=\frac{3}{7} x(\zeta) .
$$

Let $x, y \in \mathbb{R}$ and $t \in[0,1]$. Then

$$
|f(t, x)-f(t, y)| \leq \frac{e^{-\pi t}}{\left(25 \sqrt{\pi}+e^{-\pi t}\right)}|x-y| \leq \frac{1}{25 \sqrt{\pi}+e^{-\pi}}|x-y|
$$

Hence the condition $\left(H_{1}\right)$ holds with $\omega=\frac{1}{25 \sqrt{\pi}+e^{-\pi}}$. Also, for $x, y \in C[0,1]$, we have

$$
|g(t, x)-g(t, y)| \leq \frac{1}{19}|x-y| .
$$

Hence $\left(H_{2}\right)$ is satisfied with $\varpi=\frac{1}{19}$. We can find that

$$
\begin{aligned}
& \Delta \quad: \quad=\frac{\Gamma(\beta+p+1) \Gamma(\beta+1)}{\Gamma(\beta+p+1) T^{\beta}-\Gamma(\beta+1) \theta \eta^{\beta+p}}=0.91716, \\
& \Lambda \quad: \quad=\frac{T^{\alpha+\beta}}{\Gamma(\alpha+\beta+1)}+\frac{T^{\alpha+\beta+\sigma}}{\Gamma(\alpha+\beta+\sigma+1)}+\frac{|\Delta| T^{\beta}}{\Gamma(\beta+1)}\left(\frac{T^{\alpha+\beta}}{\Gamma(\alpha+\beta+1)}\right. \\
&\left.+\frac{T^{\alpha+\beta+\sigma}}{\Gamma(\alpha+\beta+\sigma+1)}+\frac{|\theta| \eta^{\alpha+\beta+p}}{\Gamma(\alpha+\beta+p+1)}+\frac{|\theta| \eta^{\alpha+\beta+p+\sigma}}{\Gamma(\alpha+\beta+p+\sigma+1)}\right)=2.2221, \\
& \Lambda_{1} \quad: \quad=\frac{\left|\Delta\left(\theta \eta^{p}-\Gamma(p+1)\right)\right| T^{\beta}}{\Gamma(p+1) \Gamma(\beta+1)}+1=1.92083, \\
& \Lambda_{2} \quad: \quad=\frac{|\lambda| T^{\beta}}{\Gamma(\beta+1)}\left(1+\frac{|\Delta| T^{\beta}}{\Gamma(\beta+1)}+\frac{|\Delta \theta| \eta^{\beta+p}}{\Gamma(\beta+p+1)}\right)=0.35033 .
\end{aligned}
$$

Therefore, we have

$$
\Lambda \omega+\Lambda_{1} \varpi<1-\Lambda_{2} .
$$

Hence, by Theorem 6 , the fractional boundary value problem (4.1) has a unique solution on $[0,1]$.

Example 4.2. Consider the following fractional boundary value problem:

$$
\left\{\begin{array}{l}
D^{\frac{3}{7}}\left(D^{\frac{1}{2 e}}+\frac{1}{17}\right) x(t)=\frac{\tanh \left(t+e^{1-t}\right) \sqrt{(1+t)} \sin \left(x^{2}-1\right)}{e^{\frac{1+3 t}{5}}\left(|x|+t^{2}+1\right)} \\
+\int_{0}^{t} \frac{(t-s)^{\frac{e^{2}}{3}}-1}{\Gamma\left(\frac{e^{2}}{3}\right)}\left(\frac{\tanh \left(t+e^{1-t}\right) \sqrt{(1+t)} \sin \left(x^{2}(s)-1\right)}{25 e^{\frac{1+3 t}{5}}\left(|x(s)|+t^{2}+1\right)}\right) d s, t \in[0,1], \\
x(0)=\frac{1}{9}+\frac{\ln 3}{11} x(\xi), x(1)=\frac{1}{10} \int_{0}^{\frac{2}{7}} \frac{\left(\frac{2}{7}-s\right) \frac{1}{7}}{\Gamma\left(\frac{8}{7}\right)} d s, 0<\xi<1,
\end{array}\right.
$$

with $\alpha=\frac{3}{7}, \beta=\frac{1}{2 e}, \lambda=\frac{1}{17}, \sigma=\frac{e^{2}}{3}, x_{0}=\frac{1}{9}, \theta=\frac{1}{10}, p=\frac{8}{7}, \eta=\frac{2}{7}$ and $f(t, x)=$ $\frac{\tanh \left(t+e^{1-t}\right) \sqrt{(1+t)} \sin \left(x^{2}-1\right)}{25 e^{\frac{1+3 t}{5}}\left(|x|+t^{2}+1\right)}, g(x)=\frac{\ln 3}{11} x(\zeta)$. 
For any $x, y \in C[0,1]$, we have

$$
|g(x)-g(y)| \leq \frac{\ln 3}{11}\|x-y\|,
$$

which implies that the function $g(x)=\frac{\ln 3}{11} x(\zeta)$ is contractive. Moreover, $g(0)=0$. Hence, the condition $\left(H_{3}\right)$ is satisfied. Also for $x, y \in$ and $t \in[0,1]$, we have

$$
|f(t, x)| \leq \frac{\tanh \left(t+e^{1-t}\right) \sqrt{(1+t)}}{25 e^{\frac{1+3 t}{5}}}(|x|-1) .
$$

So, we take $\gamma(t)=\frac{\tanh \left(t+e^{1-t}\right) \sqrt{(1+t)}}{25 e^{\frac{1+3 t}{5}}}$ and $\psi(|x|)=|x|+1$, then the condition $\left(H_{4}\right)$ is satisfied. With the given values, it is found that

$$
\begin{aligned}
&\|\gamma\|= 4.5912 \times 10^{-2} \\
& \rho \quad: \quad= {\left[\frac{T^{\alpha+\beta}}{\Gamma(\alpha+\beta+1)}+\frac{T^{\alpha+\beta+\sigma}}{\Gamma(\alpha+\beta+\sigma+1)}+\frac{|\Delta| T^{\beta}}{\Gamma(\beta+1)}\left(\frac{T^{\alpha+\beta}}{\Gamma(\alpha+\beta+1)}\right.\right.} \\
&\left.\left.+\frac{T^{\alpha+\beta+\sigma}}{\Gamma(\alpha+\beta+\sigma+1)}+\frac{|\theta| \eta^{\alpha+\beta+p}}{\Gamma(\alpha+\beta+p+1)}+\frac{|\theta| \eta^{\alpha+\beta+p+\sigma}}{\Gamma(\alpha+\beta+p+\sigma+1)}\right)\right]\|\gamma\|=0.13510 \\
& \Delta \quad: \quad= \frac{\Gamma(\beta+p+1) \Gamma(\beta+1)}{\Gamma(\beta+p+1) T^{\beta}-\Gamma(\beta+1) \theta \eta^{\beta+p}}=0.93641 \\
& \Lambda \quad: \quad= \frac{T^{\alpha+\beta}}{\Gamma(\alpha+\beta+1)}+\frac{T^{\alpha+\beta+\sigma}}{\Gamma(\alpha+\beta+\sigma+1)}+\frac{|\Delta| T^{\beta}}{\Gamma(\beta+1)}\left[\frac{T^{\alpha+\beta}}{\Gamma(\alpha+\beta+1)}\right. \\
& \quad\left.+\frac{T^{\alpha+\beta+\sigma}}{\Gamma(\alpha+\beta+\sigma+1)}+\frac{|\theta| \eta^{\alpha+\beta+p}}{\Gamma(\alpha+\beta+p+1)}+\frac{|\theta| \eta^{\alpha+\beta+p+\sigma}}{\Gamma(\alpha+\beta+p+\sigma+1)}\right]=2.5636 \\
& \Lambda_{1} \quad: \quad=\frac{\left|\Delta\left(\theta \eta^{p}-\Gamma(p+1)\right)\right| T^{\beta}}{\Gamma(p+1) \Gamma(\beta+1)}+1=1.99230 \\
& \Lambda_{2} \quad: \quad=\frac{|\lambda| T^{\beta}}{\Gamma(\beta+1)}\left(1+\frac{|\Delta| T^{\beta}}{\Gamma(\beta+1)}+\frac{|\Delta \theta| \eta^{\beta+p}}{\Gamma(\beta+p+1)}\right)=0.12943 .
\end{aligned}
$$

and the condition

$$
\frac{\delta_{0}}{\Lambda_{1}\left|x_{0}\right|+\rho \psi\left(\delta_{0}\right)}>\frac{1}{1-\left(\Lambda_{1} \varpi+\Lambda_{2}\right)}
$$

implies that $\delta_{0}>0.62271$. Clearly all the conditions of Theorem 10 are satisfied. Hence by the conclusion of Theorem 10, the fractional boundary value problem (4.2) has a solution on $[0,1]$.

Example 4.3. Consider:

$$
\left\{\begin{array}{l}
D^{\frac{2}{3}}\left(D^{\frac{5}{6}}+\frac{2}{19}\right) x(t)=\frac{1}{23(\ln (t+1)+1)}\left(\sinh t+\frac{|x(t)|}{1+|x(t)|}+|x(t)|\right)+1+\ln (t+3) \\
+\int_{0}^{t} \frac{(t-s)^{\frac{1}{3}}}{\Gamma\left(\frac{4}{3}\right)}\left(\frac{1}{23(\ln (t+1)+1)}\left(\sinh t+\frac{|x(s)|}{1+|x(s)|}+|x(s)|\right)+1+\ln (s+3)\right) d s, t \in[0,1], \\
x(0)=\sum_{i=1}^{n} c_{i} \frac{\left|x\left(t_{i}\right)\right|}{1+\left|x\left(t_{i}\right)\right|}, x(1)=\sqrt{2} \int_{0}^{\frac{1}{3}} \frac{\left(\frac{1}{3}-s\right)^{\frac{1}{6}}}{\Gamma\left(\frac{7}{6}\right)} d s .
\end{array}\right.
$$

where $0<t_{1}<t_{2}<\ldots<t_{n}<1, c_{i}, i=1,2, \ldots, n$, are given positive constants with $\sum_{i=1}^{n} c_{i}<\frac{1}{2}$.

Consider the fractional boundary value problem (4.3), with, $\alpha=\frac{3}{2}, \beta=\frac{5}{6}, \lambda=$ $\frac{2}{19}, \sigma=\frac{4}{3}, \theta=\sqrt{2}, p=\frac{7}{6}, \eta=\frac{1}{3}$ and $f(t, x)=\frac{e^{-\pi t}|x(t)|}{\left(25 \sqrt{\pi}+e^{-\pi t}\right)(1+|x(t)|)}+\frac{1}{2}+\cosh \left(t^{2}+2\right)$, $g(x)=\sum_{i=1}^{n} c_{i} \frac{\left|x\left(t_{i}\right)\right|}{1+\left|x\left(t_{i}\right)\right|}$. 
Let $t \in[0,1]$ and $x, y \in \mathbb{R}$. Then

$$
|f(t, x)-f(t, y)| \leq\left|\frac{1}{23(\ln (t+1)+1)}\right||x-y| \leq \frac{1}{23}|x-y| .
$$

Hence the condition $\left(H_{1}\right)$ holds with $\omega=\frac{1}{23}$. Also, for any $x, y \in C([0,1])$, we have

$$
|g(x)-g(y)| \leq \sum_{i=1}^{n} c_{i}|x-y| .
$$

So, $\left(H_{2}\right)$ is satisfied with $\varpi=\sum_{i=1}^{n} c_{i}<\frac{1}{2}$.

Thus the condition

$$
\omega=4.3478 \times 10^{-2}<\frac{\Gamma(\sigma+1)}{\left[\Gamma(\sigma+1)+T^{\sigma}\right]}=0.5435 .
$$

is satisfied. It follows from Theorem 8 that the fractional boundary value problem (4.3) has a unique solution on $[0,1]$, and from Theorem 13 , the fractional boundary value problem (4.3) has the Ulam-Hyers stability.

\section{R E F E R E N C E S}

1. S. Belarbi and Z. Dahmani: Some applications of Banach fixed point and Leray Schauder theorems for fractional boundary value problems. Journal of Dynamical Systems and Geometric Theories. 11(1-2), 2013, 53-79.

2. M. Benchohra, S. Hamani and S.K. Ntouyas: Boundary value problems for differential equations with fractional order and nonlocal conditions. Nonlinear Anal. 71, 2009, 23912396.

3. R. Ben Taher, M. EL Fetnassi and M. Rachidi: On the stability of some rational difference equations and Ostrowski conditions. Journal of Interdisciplinary Mathematics. 16(1), 2013, pp. 19-36.

4. A. V. Bitsadze: On the theory of nonlocal boundary value problems. Dokl. Akad. Nauk SSSR. 277, 1984, 17-19.

5. L. Byszewski: Theorems about existence and uniqueness of solutions of a semilinear evolution nonlocal Cauchy problem. J. Math. Anal. Appl. 162, 1991, 494-505.

6. M. Houas: Existence of solutions for fractional differential equations involving two Riemann-Liouville fractional orders. Anal. Theory Appl. 34 (3)2018, 253-274.

7. M. Houas, Z. Dahmani and M. Benbachir: New results for a boundary value problem for differential equations of arbitrary order. International Journal of Modern Mathematical Sciences. 7(2), 2013, 195-211.

8. M. Houas and Z. Dahmani: New results for multi-point boundary value problems involving a sequence of Caputo fractional derivatives. Electronic Journal of Mathematics and its Applications. 1 (2), 2015, 48-62.

9. M. Houas and Z. Dahmani: On existence of solutions for fractional differential equations with nonlocal multi-point boundary conditions. Lobachevskii Journal of Mathematics. 37(2), 2016, 120-127. 
10. A.A. Kilbas and S.A. Marzan: Nonlinear differential equation with the Caputo fraction derivative in the space of continuously differentiable functions. Differential Equations. 41(1), 2005, 84-89.

11. V. Lakshmikanthan and A.S. Vatsala: Basic theory of fractional differential equations. Nonlinear Anal. 69(8), 2008, 2677-2682.

12. N. Lungu and D. Popa: Hyers-Ulam stability of a first order partial di erential equation. J. Math. Anal. Appl. 385, 2012, 86-91.

13. F. Mainardi: Fractional calculus, Some basic problem in continuum and statistical mechanics. Fractals and fractional calculus in continuum mechanics. Springer, Vienna. 1997.

14. S K. Ntouyas: boundary value problems for nonlinear fractional differential equations and inclusions with nonlocal and fractional integral boundary conditions. Opuscula Math. 33(1), 2013, 117-138.

15. D. O'Regan: Fixed-point theory for the sum of two operators. Appl. Math. Lett. 9, $19961-8$.

16. I. A. Rus: Ulam stabilities of ordinary differential equations in a Banach space. Carpathian J. Math. 26, 2010, 103-107.

17. S.M. Ulam: A collection of mathematical problems. Interscience, New York. 1968.

18. Z. Wei, C. Pang and Y. Ding: Positive solutions of singular Caputo fractional differential equations with integral boundary conditions. Commun. Nonlinear Sci. Numer. Simul. 17, 2012, 3148-3160.

19. J.R Wang and Z. Lin: Ulam's type stability of Hadamard type fractional integral equations. Filomat. 28(7), 2014, 1323-1331.

20. J.R. Wang, M. Feckan and Y. Zhou: Ulam's type stability of impulsive ordinary differential equations. J. Math. Anal. Appl. 395, 2012, 258-264.

21. J.R. Wang, L. Lv and Y. Zhou: Ulam stability and data dependence for fractional differential equations with Caputo derivative. Electronic Journal of Qualitative Theory of Differential Equations. 63, 2011, 1-10.

22. J.R. Wang and X. Li: Ulam-Hyers stability of fractional Langevin equations. Applied Mathematics and Computation. 258, 2015, 72-83.

23. R.Yan, S. Sun, Y. Sun and Z. Han: Boundary value problems for fractional differential equations with nonlocal boundary conditions. Advances in Difference Equations. 2013:176, 2013, 1-12.

24. W. Zhon and W. Lin: Nonlocal and multiple-point boundary value problem for fractional differential equations. Computers and Mathematics with Applications. 59(3), 2010, 1345-1351.

25. J. Zhao, P. Wang and W. Ge: Existence and nonexistence of positive solutions for a class of third order BVP with integral boundary conditions in Banach spaces. Commun. Nonlinear Sci. Numer. Simul. 16, 2011, 402-413. 
Houas Mohamed

Laboratory FIMA

UDBKM, University of Khemis Miliana

44125 Khemis Miliana, Algeria

houas-mohamed@yahoo.fr

Bezziou Mohamed

Faculty SEI,

University of Mostaganem

Algeria

m.bezziou@univ-dbkm.dz 